\title{
Danninger, Gabriele
}

\section{Neurodidaktik und Lernwirksamkeit im Lernfeld Ernährung}

Haushalt in Bildung \& Forschung 5 (2016) 4, S. 25-34

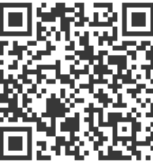

Quellenangabe/ Reference:

Danninger, Gabriele: Neurodidaktik und Lernwirksamkeit im Lernfeld Ernährung - In: Haushalt in Bildung \& Forschung 5 (2016) 4, S. 25-34 - URN: urn:nbn:de:0111-pedocs-203371 - DOI: 10.25656/01:20337

https://nbn-resolving.org/urn:nbn:de:0111-pedocs-203371 https://doi.org/10.25656/01:20337

in Kooperation mit / in cooperation with:

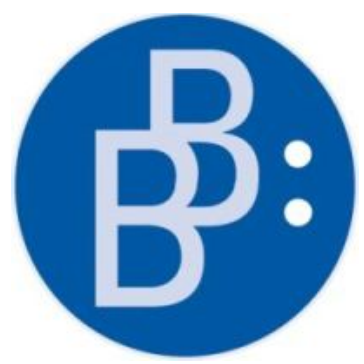

https://www.budrich.de

\section{Nutzungsbedingungen}

Gewährt wird ein nicht exklusives, nicht übertragbares, persönliches und beschränktes Recht auf Nutzung dieses Dokuments. Dieses Dokument ist ausschließlich für den persönlichen, nicht-kommerziellen Gebrauch bestimmt. Die Nutzung stellt keine Übertragung des Eigentumsrechts an diesem Dokument dar und gilt vorbehaltlich der folgenden Einschränkungen: Auf sämtlichen Kopien dieses Dokuments müssen alle Urheberrechtshinweise und sonstigen Hinweise auf gesetzlichen Schutz beibehalten werden. Sie dürfen dieses Dokument nicht in irgendeiner Weise abändern, noch dürfen Sie dieses Dokument für öffentliche oder kommerzielle Zwecke vervielfältigen, öffentlich ausstellen, aufführen, vertreiben oder anderweitig nutzen.

Mit der Verwendung dieses Dokuments erkennen Sie die Nutzungsbedingungen an.

\section{Terms of use}

We grant a non-exclusive, non-transferable, individual and limited right to using this document.

This document is solely intended for your personal, non-commercial use. Use of this document does not include any transfer of property rights and it is conditional to the following limitations: All of the copies of this documents must retain all copyright information and other information regarding legal protection. You are not allowed to alter this document in any way, to copy it for public or commercial purposes, to exhibit the document in public, to perform, distribute or otherwise use the document in public.

By using this particular document, you accept the above-stated conditions of use.

\section{Kontakt / Contact:}

\section{peDOCS}

DIPF | Leibniz-Institut für Bildungsforschung und Bildungsinformation Informationszentrum (IZ) Bildung

E-Mail:pedocs@dipf.de

Internet: www.pedocs.de

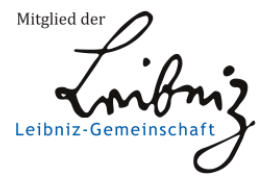




\section{Jahrgang} Heft 4

2016

旁

๓

$\bullet$

号

$\bullet$

돈

용

『্冖

$\frac{\bar{T}}{\Phi}$

$\frac{9}{2}$

정

은

$\stackrel{ \pm}{\frac{1}{2}}$

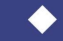

c

$\frac{0}{1}$

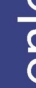

$\xi$

ह

N

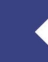

$=$

s

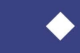

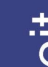

$\frac{2}{2}$

항

ISSN 2193-8806

\section{Haushalt in \\ Forschung}

\section{Lernwirksamkeitsmessung I}

Theoriebildung \& Messinstrumente
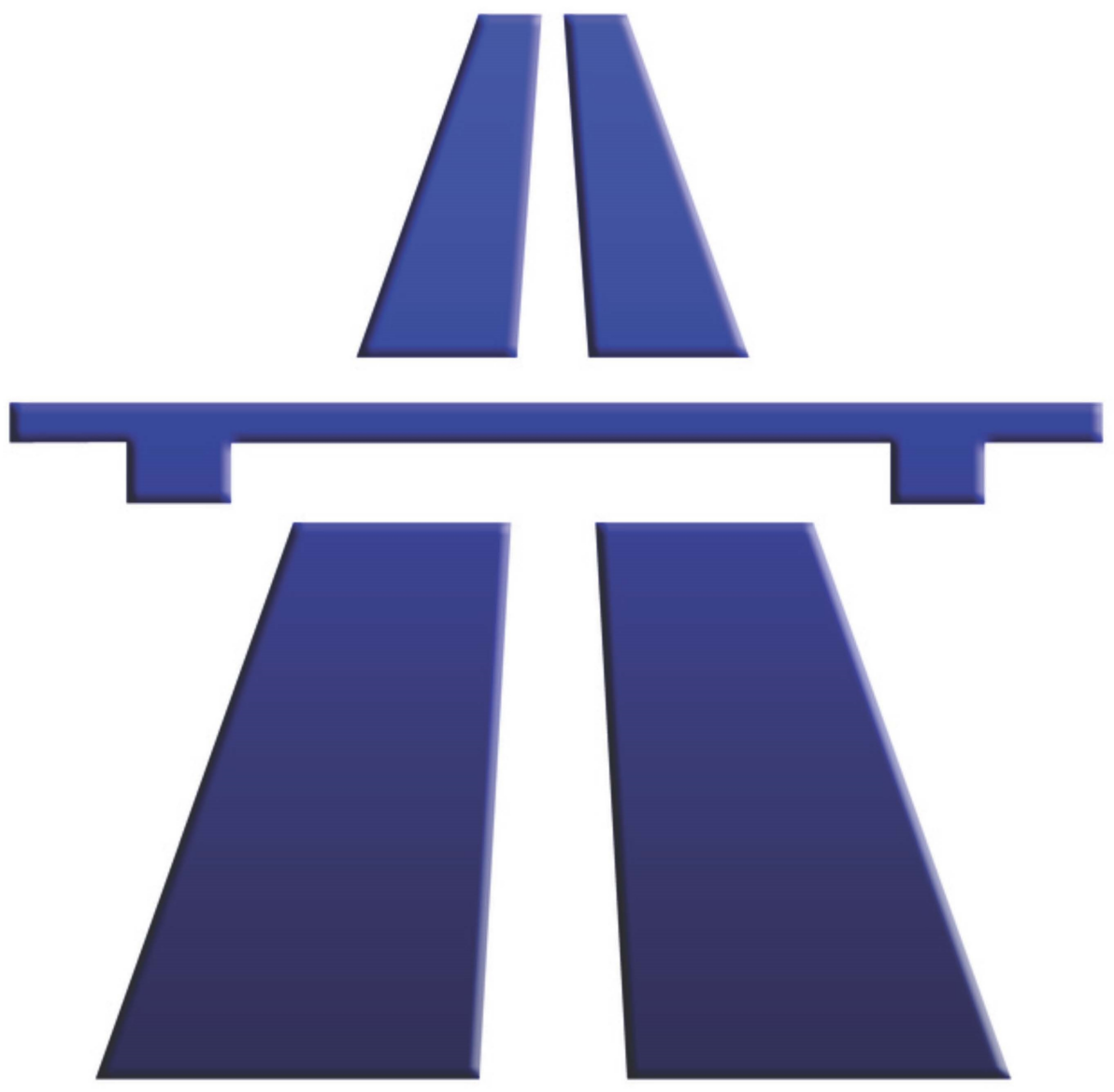


\section{Inhaltsverzeichnis}

Ursula Buchner

Editorial. 2

Werner Brandl

Lernstand erheben, Lernwirksamkeit feststellen,

Lernleistung beurteilen - eine Skizze des Bezugssystems

Gabriele Danninger

Neurodidaktik und Lernwirksamkeit im Lernfeld Ernährung.... 25

Sabine Albert

Die Bedeutung der reflexiven Selbstforschung für die Professionalisierung von Lehrpersonen

Sandro Biaggi \& Claudia Wespi

Professionskompetenzen fördern mit eigenen Videos aus dem Praktikum -

Einblicke in Erfahrungen von Studierenden und Dozierenden

Irmgard Dachtler-Freiler

Neuer Lehrplan - und was nun? Basisüberlegungen zur Lernwirksamkeit des neuen Lehrplans 61

Martina Überall, Maria Lerchbaumer, Christa Meliss \& Birgit Wild

Nachhaltigkeit schmeckt!

Ursula Buchner \& Maria Magdalena Fritz

Lernen im Fach Gesundheit und Soziales: Was wirkt?

Karin Lindner

Langfristige Auswirkungen von Auslandspraktika

auf die berufliche Entwicklung.

Julia Hirsch

Rezension: Essen und Ernährungsbildung in der KiTa 123 
Neurodidaktik und Lernwirksamkeit |

\section{Gabriele Danninger}

\section{Neurodidaktik und Lernwirksamkeit im Lernfeld Ernährung}

Neurowissenschaftliche Perspektiven in der Lehr- und Unterrichtsforschung in Form von gehirngerechtem Lehren und Lernen haben in den letzten Jahrzehnten neue Impulse für den Unterricht gefördert (Luttenberger, 2014). Das Lernen von Entscheidungen im Bildungsprozess, das dem Alltagshandeln zugrunde liegt, wird aus neurodidaktischer Sicht in den Fokus gerückt, um effektive und nachhaltige Lernergebnisse zu sichern.

Schlüsselwörter: Neurodidaktik, gehirngerechtes Lehren und Lernen, Konstruktivismus, Lernwirksamkeit

\section{Neurodidaktische Sichtweisen und Perspektiven}

Unser Gehirn lernt immer. Es tut nichts lieber und kann gar nicht anders. Ein Leben lang. (Spitzer, 2004, S. 8)

Die Neurodidaktik stellt eine Schnittstelle zwischen den kognitiven Neurowissenschaften und der Didaktik dar. Ihre Aufgabe besteht darin, die Erkenntnisse der Gehirnforschung in Bezug auf Lehren und Lernen für die Umsetzung im Unterricht nutzbar zu machen. In der traditionellen Auffassung wird unter Didaktik die "Begründung der Auswahl und Anordnung der Lehrinhalte und Methodik als Lehre von den Lehr-Lern-Verfahren" (Herrmann, 2009, S. 9) unterschieden. In der neurodidaktischen Sichtweise werden Voraussetzungen, Strukturen, Prozesse und Ergebnisse des Lernens aufgrund neurowissenschaftlicher Einsichten interpretiert und modifiziert (Gasser, 2010; Herrmann, 2009). Lernen beruht auf Selbsttätigkeit, Selbständigkeit und Selbstverantworlichkeit und bedarf eines Wechsels von Anspannung und Entspannung. Probieren und Experimentieren stehen dabei im Zentrum (Herrmann, 2009).

Von der neurowissenschaftlichen Forschung werden diese pädagogisch relevanten Prozesse begründet. Gehirne verarbeiten Sinneseindrücke, die von den Sinnesorganen aufgenommen und in Form von Impulsmustern weitergeleitet werden und als Informationsübermittler dienen. Ein Neuron wird aktiv, als elektronische Impulse werden Informationen an den Fasern der Nervenzellen entlang und auf chemischen Wege über die Synapsen übertragen. Als Synapsen werden die Stellen, an denen die Botschaft zwischen den Nervenzellen übermittelt 


\section{Neurodidaktik und Lernwirksamkeit}

wird, bezeichnet. Die Übertragung der gleichen Impulse funktioniert aufgrund der Unterschiedlichkeit der Synapsenstärke. Die Synapsenstärke im Gehirn wird selbständig festgelegt, die Gehirnstruktur ist nicht statisch, sondern wächst ständig und gebrauchsabhängig. So lernt unser Gehirn langsam, aber zuverlässig. (Spitzer, 2004)

\subsection{Veränderung und Stabilisierung beim Lernen}

Im Gehirn laufen ständig Impulse über die Synapsen der Nervenzellen; das passiert schon im Mutterleib, wenn das Ungeborene seine Umwelt ertastet oder Geräusche hört. Wenn solche Impulse immer wieder ähnlich ablaufen, entstehen quasi Spuren, zunächst in den einfachen Arealen, dann in den komplexeren, und je öfter diese Spuren benutzt werden, umso mehr verfestigen sie sich, wie bei einem Trampelpfad im Tiefschnee. Diese Spurenbildung nennen wir Lernen. (Spitzer, 2004, S. 30)

Manfred Spitzer hat für die Veränderung beim Lernen das Bild der Entstehung und langsamen Verfestigung von Spuren im verschneiten Park gefunden. Sind einmal Spuren entstanden, sorgt das Gehirn dafür, dass sie sich verfestigen, (Spitzer, 2004) denn das Gehirn lernt das, womit es sich gerade beschäftigt. Laut Hüther stellt sich die entscheidende Frage, wie und wozu wir das Gehirn benutzen sollen, um das mögliche Potential auszuschöpfen. Dabei kommt es vor allem auf den Aufbau an und dieser ist seiner Auffassung nach davon abhängig, wofür das Gehirn bisher gebraucht wurde.

Wesentliche Erfahrungen der frühen Kindheit und im Jugendalter führen zur Stabilisierung von speziellen neuronalen Verschaltungen, die auch im späteren Leben einfach durch bestimmte Wahrnehmungen und Erlebnisse aktivierbar und bestimmend für das Denken, Fühlen und Handeln sein können (Hüther, 2009). Diese Sichtweisen zu den frühen Programmierungen sind vor allem im Lernbereich Ernährung von Bedeutung, da unbewusste Installationen und „Spuren“ bewusst gemacht und erkannt werden müssen. Die ,gesunde Jause“, wie sie bereits in Kindergärten und in der Primärstufe temporär implementiert wird, kann als Basis für die Entwicklung einer Grundhaltung der gesunden Ernährung und eines kritischen Konsumverhaltens dienen. „Ein zeitlebens lernfähiges Gehirn ist auch lebenslänglich veränderbar. Auch die, während der Phase der Hirnentwicklung möglicherweise entstandenen einseitigen, unbalancierten oder defizitären Installationen sind daher in gewissem Umfang auch noch im erwachsenen Zustand korrigierbar" (Hüther, 2009, S. 24).

\subsection{Regeln und Beispiele für unser Gehirn}

Bei Berücksichtigung der Gedächtnisspurenbildung ist wesentlich, dass sich jede einzelne Erfahrung nur geringfügig niederschlägt, während nach vielen 


\section{Neurodidaktik und Lernwirksamkeit}

Erfahrungen, Wahrnehmungs-, Denk- und Gefühlsakten die Regeln, welche hinter den einzelnen Erfahrungen erkennbar sind, in Form von festen Spuren im Gehirn festgelegt werden (vgl. Spitzer, 2008, S. 29f). Unser Gehirn lernt und strukturiert sich nach Regeln, die hinter den Einzelheiten stehen, es merkt sich nicht jede Kleinigkeit. So sollen im Unterricht die Regelhaftigkeiten, die durch Ähnlichkeiten verursacht werden, hervorgehoben werden.

Im Lernfeld Ernährung hat nach dieser Auffassung eine einzige Erfahrung noch keinen nachhaltigen Effekt, denn es wird die Allgemeinheit hinter den Kleinigkeiten wahrgenommen. Es müssen viele Erfahrungen gemacht werden, um langsam, aber effektiv zu lernen. Neuronen vermehren sich beim Lernen nicht, sondern es kommt dabei zu weiteren internen Verknüpfungen. (Blakemore \& Frith, 2006). Grundsätzlich fahren wir mit einem Autopiloten durch das Leben, viele Entscheidungen werden ohne zu langes Nachdenken getroffen und die rationale Einsicht ist dabei nicht standardmäßig vorprogrammiert. Die anregende Lernumgebung sorgt dafür, dass effiziente Lerninhalte herausgefiltert werden (Spitzer, 2004).

\subsection{Wissen konstruieren und Bedeutungen verstehen}

Der Konstruktivismus ist eine systemische Erkenntnistheorie (vgl. Glasersfeld, 1996, Foerster, 1997, Simon, 2015) und es geht um die Beschäftigung mit der Frage, wie Wirklichkeit wahrgenommen wird. Die Wahrnehmung der Wirklichkeit ist nach Auffassung der Systemtheorie perspektivisch, selektiv und nicht direkt beobachtbar. Das Gehirn erhält ,keine bedeutungshaften und verlässlichen Informationen über die Umwelt“" (Roth, 1996, S. 21), es erschafft auf Grundlage von Außenreizen und individuellen neuronalen Aktivitäten das, was wir als Informationen über unsere Welt und als Bedeutungen verstehen. „Lernen ist ein aktiver Prozess der Bedeutungserzeugung" (Markowitsch, 2002, zit. nach Roth, 2004, S. 502), welcher in den einzelnen Personen sehr unterschiedlich abläuft. So existiert kein objektiver Maßstab für die Richtigkeit dessen, was wir wahrnehmen und $\mathrm{zu}$ glauben wissen. Nach Ernst von Glasersfeld beruht der radikale Konstruktivismus auf der „Annahme, dass alles Wissen, wie immer man es auch definieren mag, nur in den Köpfen von Menschen existiert" (von Glasersfeld, 1996a, S. 22).

So sind auch bestimmte Grundannahmen des Konstruktivismus, welche sich auf Vorgänge des Wahrnehmens, Erkennens und den Wissenserwerb beziehen, für Pädagoginnen und Pädagogen von besonderer Relevanz, da sich Wissen nur auf der Basis eigener Erfahrungen erschaffen lässt. Wissen ist nach dieser Auffassung nicht übertragbar (Roth 2009, S. 58), denn jedes Lebewesen ist autonom und verfügt über ein geschlossenes System. Erkenntnisse werden somit durch eigenes Handeln aufgebaut, denn lebende Systeme arbeiten „selbstorganisiert“ und „selbstreferenziell“ und die eigene innere Struktur bestimmt die Wahrnehmung 
(Maturana \& Varela, 1990). Es existieren individuelle Wirklichkeitskonstruktionen d. h. die „Erschaffung der Mitmenschen nach unserem Bilde“ (von Glasersfeld 1996, S. 196) und verschiedene Möglichkeiten der Welterklärung.

\section{Gehirngerechtes Lehren und Lernen}

\subsection{Lernen als aktiver Prozess}

Die lernende Person übernimmt bei jedem Lernen Steuerungs- und Kontrollprozesse. Elaboriertes Lernen vollzieht sich, wenn das Individuum in einer unstrukturierten, chaotischen Situation durch subjektive Konstruktion dieser Problemstellung eine Struktur geben kann (Reich, 2000). Nach Auffassung kognitivistischkonstruktivistischer Lerntheorien verarbeiten lernende Personen Informationen aktiv und selbständig und sind nicht durch äußere Reize steuerbar. Eine Vermittlung von Wissen im engeren Sinne ist nicht möglich, womit sich auch die Rolle der Lehrerinnen und Lehrer ändert. Lernen begründet sich auf eigenständiger, individueller Auseinandersetzung mit dem Inhalt und Lernwege sind nicht planbar. Lernfördernd wirkt eine Konfrontation mit verschiedenen Perspektiven zu ein und demselben Thema. Die Lehrperson übernimmt dabei die Rolle eines Moderators.

Neues Lernen baut auf Vorwissen auf, welches grundsätzlich bestimmt, was gelernt wird. Die Bedeutung dieser bereichsspezifischen Vorkenntnisse wird von Klauer (1993) in der Metapher „Matthäus-Effekt" im Gleichnis von den Talenten abgebildet. „Denn wer da hat, dem wird gegeben, [...] wer aber nicht hat, dem wird auch das genommen, was er hat." (Matthäus Evangelium Kapitel 25, Vers 29). Der Bezug wird damit zur begünstigten Rolle der guten Vorkenntnisse und deren Vernetzung im neuen Wissenserwerb hergestellt.

Die Lernleistung setzt sich grundsätzlich aus drei Bereichen zusammen, erstens aus der individuellen Bereitschaft, d. h. der Motivation zum Lernen, zweitens der Fähigkeit (Begabung) und drittens den Möglichkeiten, Übungsanreizen, die von außen geboten werden und Vorwissen aktivieren. Je höher die Motivation und Aktivität jeder einzelnen Schülerin und jedes einzelnen Schülers, desto höher fällt die Merkleistung aus, da das Gehirn das Finden von Information als Belohnung (intrinsische Motivation) registriert. Individuelle Konstruktionsleistungen können durch Selbststeuerung und Eigenverantwortlichkeit des Lernens, Lernen durch Erleben, Bewerten des Erlebten, dem Aufbau von Erfahrung und der Interaktion mit anderen Lernenden ermöglicht werden (Spitzer, 2008; Hermann, 2009).

\subsection{Lernen reflektieren}

Die Auseinandersetzung mit der Förderung eigener Lernprozesse kann Schülerinnen und Schülern zu mehr Erfolg und Spaß am Lernen führen, sowie 


\section{Neurodidaktik und Lernwirksamkeit}

nachhaltiges Lernen ermöglichen. Reflektierende Fragen in Form von regelmäßigen Tagesrückblicken dienen zur Unterstützung von Wahrnehmungsund Aufmerksamkeitsprozessen der Lernfortschritte. Lerntagebücher bieten sich dazu als Hilfestellung in der Selbstevaluation und der Beantwortung der Frage, welche Inhalte neu und als interessante Lernanregungen aufgenommen wurden, an. Siebert schlägt die Verwendung von Lerntagebüchern in folgender Systematisierung vor (Siebert, 2009, S. 129):

- Lernthemen, Inhalte

- Lernerlebnisse, Einsichten, Ideen

- Offene Frage, unklare Begriffe

Auch für Lehrpersonen ist es empfehlenswert ein Lehr-Lerntagebuch zu verwenden, um aus diesen Reflexionen und Fragestellungen Rückschlüsse für das Unterrichtsgeschehen und profitable Unterrichtsmethoden ableiteten zu können (Siebert, 2009). Einzelarbeiten mit eigenen Recherchen können neben Gruppenarbeiten mit vorgegebenen Materialien sowie die Abhaltung von Referaten den Lehrvortrag ergänzen. So dient zum Beispiel Lernen mit Fallvignetten dazu, Situationen elaborierter zu erfassen und unterschiedliche Lösungen und Ergebnisse zu präsentieren und zu reflektieren.

\subsection{Lehren und lernen unterstützen}

Exemplarisches Lernen zählt zu den wichtigsten Strategien des Lernens, dabei sind auch die Prinzipien Organisieren und Veranschaulichen wesentlich. Wichtige Informationen sollen speziell durch Unterrichtsmaterialien gestaltet und gekennzeichnet werden, um so die Aufmerksamkeit deutlich auf die relevanten Reize zu lenken. Empfehlenswert ist dabei vor allem einen Überblick über die Themen der Stunden zu Beginn der Unterrichtseinheit zu geben, um den ,roten Faden" der Lerneinheit nachvollziehen und Inhalte geordnet abspeichern zu können. Mit Hilfe von zusammenfassenden Schaubildern kann die Verinnerlichung komplexer Sachverhalte gefördert werden, da dadurch die nachhaltige Abspeicherung der Information durch Ausbildung von semantischen Netzwerkstrukturen unterstützt wird (Schick, 2012, S. 188).

Einfaches und variiertes Wiederholen stellen basale Lernstrategien dar, die verbal oder schriftlich erfolgen können. Vor allem das Abschreiben und das Erstellen von Lernskripten kann die Abspeicherung des Wissens wirksam vorstrukturieren und die Wissensorganisation unterstützen. Das Elaborieren, d. h. das Integrieren neuer Informationen in bereits vorhandene Wissensstrukturen stellt zudem eine weitere effektivere Variante dar. Der Transfer gilt als besonders ökonomische Form der Wissensvertiefung, welche im Wesentlichen im Gebrauch 
bereits früher erworbenen Wissens bei der Konfrontation mit neuen Inhalten oder neuen Situationen entsteht (Schick, 2012, S. 188).

Als Leitprogramm hat Schachl (2005, S. 12) elf Gebote für gutes Lehren und Lernen vorgestellt:

\section{1. Überblick vor Einzelinformationen}

Das Gehirn bereitet sich bei einem Überblick und der Verwendung von Strukturen sehr gut auf die Wahrnehmung von Einzelinformationen und Details vor, da es im Vorfeld nach vorhandenen Speicherplätzen sucht und neue Speicherplätze anlegt.

\section{Transparenz der Lehr- und Lernziele}

Der Sinn des Lernens wird durch die Wozu-Frage beantwortet und dadurch die Schaltung ,,auf Empfang“ aktiviert, wodurch die Lernbereitschaft ein Wachstum erfährt.

\section{Interesse wecken}

Interesse, Neugierde, die Anknüpfung an eigene Erfahrungen sind wesentliche Voraussetzungen für positive Gefühle und stellen eine wertvolle Basis für effizientes Lernen dar.

\section{Wiederholen}

Das Zusammenfassen des Wesentlichen und das Vergleichen mit dem Überblick stellen bewährte Methoden dar, um die Nervenschaltkreise mehrmals zu betätigen und sie dadurch zu stabilisieren. Dazu können aber auch verschiedene Varianten der Wiederholung genützt werden.

\section{Mehrere Sinne ansprechen}

Die mehrfache Vernetzung und vertiefte bzw. dauerhaftere Vernetzung in den Schaltkreisen erfolgt ebenso durch das „Begreifen“ und Selbermachen.

\section{Auf die Gefühle achten}

Positive Gefühle verstärken die Informationsaufnahme ins Gedächtnis.

\section{Rückmelden}

Die ehestmögliche Rückmeldung durch Fremd- oder Selbstkontrolle, ob das Richtige gelernt wurde ist hirnbiologisch dann wesentlich, wenn der Prozess der Speicherung in den Nervenzellen noch in Bewegung und Umlernen möglich ist. Loben, Bekräftigen und Verstärken soll dabei besonders hervorgehoben werden.

\section{Pausen einlegen}

Zur Konsolidierung, d. h. Festigung soll Zeit und Ruhe gegeben sein um eine Ähnlichkeitshemmung durch die Aufnahme von neuen Informationen $\mathrm{zu}$ verhindern. 


\section{Neurodidaktik und Lernwirksamkeit}

\section{In der richtigen Reihenfolge lehren und lernen}

Durch Vorinformation und einen roten Faden, der sich auf einander aufbauende Lernschritte bezieht, wird eine sinnvolle Vernetzung und Speicherung im Gehirn gefördert und Interferenzen und Überlagerungen, welche den Lernvorgang hemmen, vermieden.

\section{Vernetzen}

Um die vernetzte Arbeit unseres Gehirns zu unterstützen ist Lernen in $\mathrm{Zu}$ sammenhängen, fächerübergreifendes und projektorientiertes Lernen wesentlich.

\section{Beachten der individuellen Begabungen}

Es ist wichtig die Stärken und Interessen der Schülerinnen und Schüler herauszufinden, um diese zu fördern (zusammengefasst nach Schachl, 2005, S. 12).

\section{Neurodidaktische Impulse für die Unterrichtspraxis}

Für gehirngerechtes Lehren und Lernen in der Unterrichtspraxis ist die Orientierung an einem bestimmten Anstrengungsniveau und die Anerkennung von Lernerfolgen wesentlich, dabei soll auch die Lernbereitschaft und das Durchhaltevermögen gegeben sein. Strukturen und Regelmäßigkeiten, sowie Einplanung von Zeit zum Wiederholen, Vertiefen und Festigen von Lerninhalten findet dabei in der Gestaltung der Lernsituationen Beachtung.

Die Lerntheorie des amerikanischen Psychologen und Pädagogen Bruner (1961/1981), die von der neurowissenschaftlichen Forschung heute Bestätigung findet, fordert die Ausrichtung des Unterrichts mit der Priorität auf grundlegende Ideen und Strukturen der Fachwissenschaft zu gestalten. Der Autor hat den Begriff „Entdeckendes Lernen“ geprägt, in welchem Lernanregungen bzw. Lernarrangements im Mittelpunkt stehen. (http://unterricht.educa.ch/de/entdeckendes-lernen)

Beim entdeckenden Lernen wird den Fragen nachgegangen, die einzelne Schülerinnen und Schüler beschäftigen und die sie verstehen wollen. „Sich auf den Weg machen, um die Dinge und Menschen um sich herum besser begreifen zu lernen“" (Zocher, 2000, S. 8). Beim entdeckenden Lernen ist vor allem die Frage- und Zielstellung wesentlich, Lernende werden dabei zu Forschenden. Eigenverantwortlichkeit und Planung stehen für Schüler/innen im Vordergrund, Lehrende werden zu Beobachter/innen, Moderator/innen und Lernbegleiter/innen. Vier Grundsäulen des entdeckenden Lernens sind für Bruner relevant:

- Die Transferförderung und Induktion, d. h. von Einzelfällen wird auf Regeln und Gemeinsamkeiten geschlossen, 
- die Problemlösefähigkeit, d. h. „die Fragestellung zu analysieren, Hypothesen zu formulieren und zu prüfen“ (Edelmann, 2000, S. 216),

- intuitives Lernen und

- die Förderung intrinsischer Motivation (zit. nach https://www.fhkiel.de/index.php?id=879).

Schirp leitet für eine erfolgreiche Unterrichtsgestaltung aus neurobiologischer Sicht mehrere Leitsätze ab (Schirp, 2006, zit. nach Luttenberger, 2014, S. 156f), die auch im Lernfeld Ernährung im Sinne der Lernwirksamkeit zum Experimentieren anregen sollen. Studierende sind aufgefordert, ihre Unterrichtskonzeptionen anhand der folgenden Items zu prüfen um die Wirksamkeit im eigenen Praxishandeln bewusst wahrzunehmen und zu reflektieren.

- Lernsituationen sollen individuelle Lernverfahren und selbständige Lernprozesse unterstützen.

- Variationsreiche Formen des Übens, der Leistungsförderung und Leistungsdarstellung sollen in den Lernarrangements abgebildet werden. Dazu sind adaptive Formen der Leistungsfeststellung und Zeitorganisation erstrebenswert.

- Kooperative und soziale Lernarrangements sollen emotionale Erfahrungen der Lernenden miteinbeziehen.

- Die Lernatmosphäre ist von gegenseitiger Wertschätzung und Ergebnisorientierung, welche positive Selbstkonzepte der Lernenden fördern, geprägt.

- Die Aufmerksamkeit soll durch Lernangebote mit gezielten Neuigkeitswerten, wie Rätsel, Überraschungen, Außergewöhnlichem, gesteigert werden.

- Der Umgang mit Prüfungs- und Leistungsanforderungen, sowie der verantwortungsvolle Umgang mit Gefühlen wird erlernt.

- Ein Angebot lernspezifischer Strukturierungshilfen soll Hilfestellung beim Aufbau „stabiler Repräsentations- und Behaltensmuster (Mindmaps, Kernsätze, Kurzmemos, einfache Strukturskizze etc.)“ (Luttenberger, 2014, S. 157) geben und die Übertragung ins Langzeitgedächtnis gewährleisten (zusammengefasst und modifiziert nach Schirp 2006, zit. nach Luttenberger, 2014, S. 156f).

Als motivierende Faktoren für den Unterricht können zusammenfassend die Interessenserfahrung und das Erleben von Bedeutsamkeit, sowie Kompetenzerleben bzw. Nützlichkeitsüberzeugung und die Erfahrung der sozialen Eingebundenheit dienen. Besonders hervorzuheben ist in diesem Zusammenhang die Verantwortung für nachhaltige Konzepte der Lebensführung (Buchner, 2015). Für die Gelingensbedingungen des Lernerfolges in der schulischen Umsetzung der Lehr- und Lern- 


\section{Neurodidaktik und Lernwirksamkeit}

forschung wird von Roth (2011, S. 313) auf die Aktivierung der Dreiecksbeziehung zwischen Psychoneurowissenschaft, Pädagogik, Didaktik und Fort- und Weiterbildung verwiesen (vgl. Luttenberger, 2014, S. 157).

\section{Literatur}

Blakemore, S.- J. \& Frith, U. (2006). Wie wir lernen. Was die Hirnforschung darüber weiß. München: DVA.

Buchner; U. (2015). Lernwege in der Schulküche. Haushalt in Bildung \& Forschung, 4(2), 58-72. http://dx.doi.org/10.3224/hibifo.v4i2.19497

Bibel (1984). Matthäus Evangelium Kapitel 25, Vers 29.

Caspary, R. (Hrsg.). (2006). Lernen und Gehirn. Der Weg zu einer neuen Pädagogik. Freiburg: Herder.

Edelmann, W. (2000). Lernpsychologie (5.Aufl.). Weinheim: Beltz PVU.

educa.unterricht. http://unterricht.educa.ch/de/entdeckendes-lernen

Fachschule Kiel. https://www.fh-kiel.de/index.php?id=8797

Foerster, H. v. et al. (1997). Einführung in den Konstruktivismus. München: Piper.

Gasser, P. (2010). Gehirngerecht lernen. Eine Lernanleitung auf neuropsychologischer Grundlage. Bern: hep verlag.

Glasersfeld, E. v. (1996). Der Radikale Konstruktivismus. Frankfurt am Main: Suhrkamp.

Herrmann, U. (Hrsg.) (2009). Neurodidaktik. Weinheim: Beltz.

Hüther, G. (2009). Bedienungsanleitung für ein menschliches Gehirn. Göttingen: Ruprecht.

Klauer, K. J. (1993). Denktraining für Jugendliche. Göttingen: Hogrefe.

Korte, M. (2011). Wie Kinder heute lernen. München: Goldmann.

Kunter, M. \& Trautwein, U. (2013). Psychologie des Unterrichts. Paderborn: Schöningh.

Luttenberger, C. (2014). Gehirngerechtes Lehren und Lernen. Neurowissenschaftliche Perspektiven. In W. Lenz, B. Pflanzl \& W. Vogel (Hrsg.) (2014). Lehren lehren. Dynamische Professionalität in der PädagogInnenbildung. Leykam: Studienreihe der Pädagogischen Hochschule Steiermark, Band 5, 148-160.

Markowitsch, H. J. (2002). Dem Gedächtnis auf der Spur. Darmstadt: Wiss. Buchges.

Maturana, H. R. \& Varela F. J. (1990). Der Baum der Erkenntnis: Die biologischen Wurzeln des menschlichen Erkennens. München: Goldmann.

Reich, K. (2000). Systemisch-konstruktivistische Pädagogik. Neuwied:

Luchterhand. 


\section{Neurodidaktik und Lernwirksamkeit}

Roth, G. (1996). Das Gehirn und seine Wirklichkeit: Kognitive Neurobiologie und ihre philosophischen Konsequenzen (10. Aufl.) Frankfurt am Main: Suhrkamp TB Wissenschaft.

Roth, G. (2011). Möglichkeiten und Grenzen von Wissensvermittlung und Wissenserwerb. In R. Caspary (Hrsg.). (2006). Lernen und Gehirn (7. Aufl). (S. 54-69). Freiburg im Breisgau: Herder.

Roth, G. (2009). Aus Sicht des Gehirns. Frankfurt: Suhrkamp Taschenbuch Wissenschaft.

Roth, G. (2004). Warum sind Lehren und Lernen so schwierig? Zeitschrift für Pädagogik, 50(4), 496-506.

Schachl, H. (2005). Was haben wir im Kopf. Linz: Veritas.

Schick, H. (2012). Entwicklungspsychologie der Kindheit und Jugend. Stuttgart: Kohlhammer.

Schirp, H. (2006). Wie lernt unser Gehirn Werte und Orientierungen? Caspary, R. (Hrsg.). (2006). Lernen und Gehirn (7. Aufl). Freiburg im Breisgau: Herder.

Siebert, H. (2009). Selbstgesteuertes Lernen und Lernberatung (3.Aufl.). Augsburg: Ziel.

Simon, F.B. (2015) Einführung in Systemtheorie und Konstruktivismus (7.Aufl.). Heidelberg: Auer.

Spitzer, M. (2009). Lernen. Heidelberg: Spektrum.

Spitzer, M. (2008). Selbstbestimmen. Gehirnforschung und die Frage Was sollen wir tun? Heidelberg: Springer.

Spitzer, M. (2004). Wie lernt das Gehirn? Vortrag; http://www.lichterlsb.e/media/e085999f82f51a2effff807cffffffef.pdf

Zocher, U. (2000). Entdeckendes Lernen lernen. Donauwörth: Auer.

\section{Verfasserin}

Prof $^{\text {in }}$. Dr ${ }^{\text {in }}$. Mag ${ }^{\text {a }}$. Gabriele Danninger, MSc

Institut für Bildungswissenschaft und Forschung

Pädagogische Hochschule Salzburg Stefan Zweig

Akademiestraße 23

A-5020 Salzburg

E-Mail: g.danninger@gmx.at 